\title{
Bedeutung und Wandel von Leitbildern
}

\author{
Folgerungen aus der „Metropolisierung“ Shanghais 1927-2002
}

\section{The Importance and Development of "Visions"}

Conclusions from the "metropolisation" of Shanghai between 1927 and 2002

\section{Kurzfassung}

Es sind umfassende Tendenzen wie Globalisierung und Utilitarismus, die allerorts die Rahmenbedingungen der heutigen Stadtentwicklung dominieren; Utopien und hochfliegende Visionen stehen da nicht eben hoch im Kurs. Gleichwohl braucht räumliche Planung eine hinreichend konkrete Vorstellung dessen, was sie anzielt und bewirken will. Am Beispiel der größten und ökonomisch potentesten Metropole Chinas wird synoptisch für das letzte drei viertel Jahrhundert untersucht, in welchem Verhältnis die tatsächliche siedlungsstrukturelle Entwicklung und die jeweils zu Grunde gelegten Leitbilder standen bzw. stehen.

\begin{abstract}
It is such all-embracing trends as "globalisation" and "utilitarianism" which shape the dominant framework conditions for urban development today throughout the world; there is comparatively little interest in utopias or high-flown visions. And yet spatial planning does require suitably concrete visions of what it aspires to achieve and to effect. Taking as a suitable case for study the largest and economically most potent metropolis in China, this article provides a synopsis of three quarters of a century of development in examining the relationship between actual developments in settlement structure and the visions on which it was-and is-based.
\end{abstract}

Seit Mitte der 90er Jahre lässt sich eine kleine Renaissance der Leitbilder in der Raumplanung beobachten. ${ }^{1}$ Das deutet ein aktuelles Verlangen nach Orientierung an, werden damit doch Antworten gesucht auf gesellschaftliche Entwicklungen, die als problematisch empfunden werden: Das Leitbild der „sozialen Stadt" reagiert auf Segregations- und Spaltungstendenzen, „Nutzungsmischung“ auf funktionale Isolation, die „Stadt der kurzen Wege“ auf das alltägliche verkehrliche Ungemach, „nachhaltige Stadtentwicklung“ auf das systematische Ignorieren ökologischer Belange, das Bekenntnis zur „europäischen Stadt“ auf die Wucherungen von Suburbia usw. Was bedeuten nun solche Leitbilder für eine zeitgemäße, d. h. reflexive Herangehensweise an Planung?
In Näherung an eine Antwort soll die Aufmerksamkeit auf ein Fallbeispiel gerichtet werden, das zwar geographisch - fern liegen mag, gerade deswegen aber gewisse Tendenzen und Wechselwirkungen umso deutlicher offenbart. Anhand einiger Etappenbausteine wird eine knappe Skizze der jüngeren Shanghaier Stadtentwicklung gegeben, die zugleich auch das Feld der zugrunde liegenden Leitvorstellungen der Planungs-Profession mit umreißt. In einem Bogenschlag geht es dann zurück ins Hier und Heute: Steht doch Shanghai pars pro toto für das Wechselspiel von Theorie und Praxis, für das Verhältnis von Planung und Gesellschaft. 


\section{Shanghai: Stadtentwicklung und Leitbildorientierung}

Das chinesische Shanghai, d.h. der nicht von den Kolonialmächten in ihren diversen Settlements kontrollierte Bereich, verfügte zu Beginn des 20. Jahrhunderts über keine städtische Selbstverwaltung. ${ }^{2}$ Eine solch kommunale Autonomie gehörte weder zu den seinerzeitigen Traditionsbeständen Chinas noch gab es die Voraus- bzw. Einsicht in die Notwendigkeit einer modernen urbanen Infrastruktur. Als mit dem politischen Umbruch der 20er Jahre die Karten für die Regie über die Stadt neu gemischt wurden, machte man sich auf chinesischer Seite zunehmend Gedanken über ein ideales Shanghai. Entwickelt wurde eine Art Vision, die vor allem auf die koloniale Realität reagierte: Angestrebt wurde eine eigene, zusätzliche Stadt, die perspektivisch die bestehende - und fremdverwaltete - sich einverleibt.

Der „Greater Shanghai Plan“ von 1927 bzw. 1929 war ein erster Versuch, das chinesische Erfahrungsfeld der Urbanistik zu justieren; er fußte auf einer zentralen Voraussetzung: Nationalistische Truppen besetzten Shanghai im März 1927, und vier Monate später erließ die zentrale Guomindang-Regierung einen Akt, der die Selbstverwaltung der Stadt verbriefte. Gleichsam als eine Gegenoffensive zu den von den Europäern dominierten Aktivitäten im Westen der Stadt wurde von der chinesischen Administration ein umfassender Plan zur konzeptionellen (Neu-)Gliederung Groß-Shanghais lanciert. Dessen funktional vielleicht bedeutendstes Element war die Verlagerung und der projektierte Ausbau des Hafens in der Gegend von Wusong - ein Vorhaben, das bereits ein strategisches Hauptkapitel des "Industialisierungs-Plans“ (1918-1921) von Sun Yatsen gewesen war und nun wiederaufgelegt wurde. Daneben war - Veerkehr und Transport stellten offensichtlich prononcierte Zielsetzungen der Modernisierung dar - ein künftiger Zentralbahnhof Bestandteil des forcierten Konzeptes. Am zielstrebigsten wurde indes eine dritte Komponente verfolgt: der Bau eines imposanten neuen Zentrums als Standort für die wichtigsten kommunalen Einrichtungen. ${ }^{3}$

Obgleich als traditionelle städtebauliche Figur des 19. Jahrhunderts auftretend ${ }^{4}$, handelt es sich hierbei um eine so ambitionierte wie moderne Planung. Und sie gibt einen Hinweis auf die prägenden Vorbilder, z.B. die Weltausstellung in Chicago 1893, die dann wiederum den Städtebau der USA bis in die 30er Jahre beeinflusste und dem City Beautiful Movement Auftrieb gab.

Doch Bürgerkrieg und die japanische Besetzung machten eine weitere Realisierung des Greater Shanghai Plan zunichte.

Als am 1. Oktober 1949 die VR ausgerufen wurde, stand in Shanghai weder die bruchlose Weiterführung der Vorkriegs-Planungen noch eine metropolitane Neugestaltung ernsthaft zur Diskussion. Das „Knowhow“, welches sich einst im "Vertragshafen“ versammelt hatte, war entschwunden: In den ersten Jahren nach der Befreiung hatten alle stadtbezogenen Arbeiten ohnedies einen restaurativen Charakter; angesichts der desolaten Nachkriegssituation konnte lediglich auf die dringlichsten und unmittelbarsten

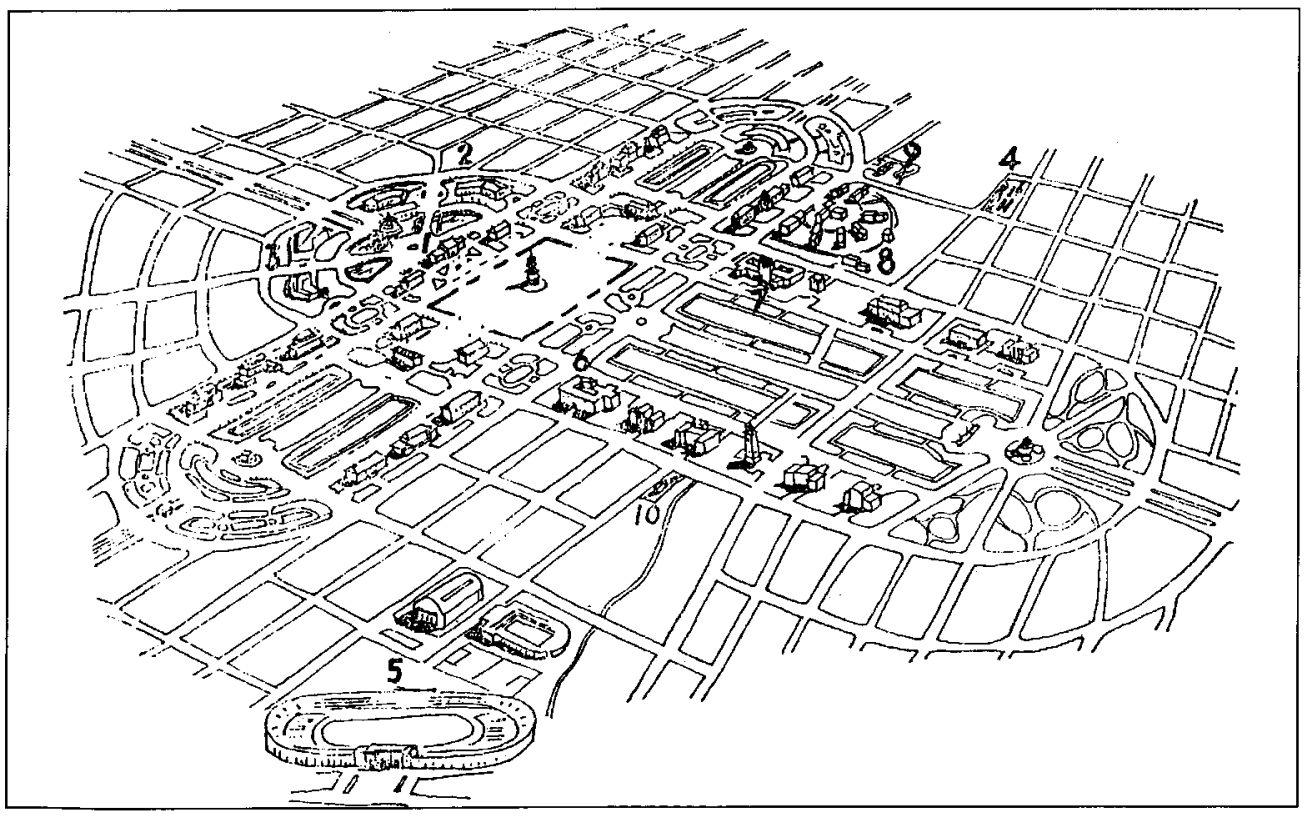

Planung für ein neues Stadtzentrum für Shanghai in Jiangwan, 1929 (Entwurf: Dong Dayou u.a.) 
Erfordernisse reagiert werden. Die Beseitigung von Missständen war das Ziel: remediale Maßnahmen in den Slumgebieten, die vor allem die Kanalisierung und Entwässerung regeln sollten, um die Seuchengefahr zu bannen, außerdem Schutz- und Drainagebauten gegen die ständige Hochwassergefahr sowie Pflanzaktionen (Platanen) in den Straßen zur Verbesserung des Mikroklimas.

\section{Ziele und Wege der Planung}

Erst in den Jahren 1952/53 etablierte sich Planung als Profession: Die politische Forderung an sie bestand darin, eine schnelle Antwort auf die moderne Industrialisierung $\mathrm{zu}$ formulieren. Bis $1956 \mathrm{lag}$ der Brennpunkt aller stadtplanerischer Tätigkeit bei den so genannten „Schlüssel- oder Schwerpunktstädten“. Als Modell aus der Sowjetunion importiert, bildeten sie das Kernstück des damaligen wirtschaftlichen Entwicklungskonzepts. Mit dem Aufbau großindustrieller Zentren im Landesinneren sollte die aus der Kolonialzeit geerbte ungleiche Verteilung von Produktionsstandorten ausgesteuert und die Entwicklung küstenferner Provinzen begünstigt werden. Im Grunde war das allerdings kaum mehr als eine auf die Bedürfnisse der Industrie abgestellte Standortwahl und Flächennutzungsplanung. ${ }^{5}$

Mit kritischer Distanz gesehen, schimmert hier die Christaller'sche Theorie der zentralen Orte durch, die eine so grundlegende wie fatale Rolle spielte bei Himmlers planmäßiger „Kolonisierung des Ostraums“. In modifizierter Form kennen wir das heute als Konzept der „dezentralen Konzentration".

Entscheidend für die weitere Entwicklung war das leise politische Eingeständnis in den Jahren 19551957, dass die Potentiale der alten Küstenzentren für den Aufbau Chinas wohl doch unverzichtbar seien. Deren industrielle Basen zu erweitern war nunmehr eine von der KP Chinas auferlegte Pflicht. Die damit angezeigten expansiven Investitionen in den Industriesektor dieser Großstädte mussten folglich via Neulanderschließung am Stadtrand erfolgen. Das aber war, bedingt durch die zu schaffende Versorgungsstruktur, teuer und, wegen des nicht minder dringenden Bedarfs an stadtnahem Ackerland, problematisch. Unter dem Druck der Verhältnisse beschritt man einfache, jedoch bedenkliche Wege: So entstand jene „widersprüchliche Mischung aus Vernachlässigung und Überbelastung der Infrastrukturen zu Gunsten industrieller Expansion auf der einen Seite, und dem Versuch, durch Mobilisierung der Bevölkerung die Industrialisierung insgesamt zu intensivieren auf der anderen" ${ }^{6}$
Shanghai war allerdings zu diesem Zeitpunkt planerisch bereits so vernachlässigt, dass es eine Art Befreiungsschlag brauchte. Den hat die Stadtregierung in den Jahren zwischen 1958 bis 1961 tatsächlich geführt, und zwar mit einer zweigleisigen Strategie.

Zum einen wurde, in enger Abstimmung mit der Zentralregierung in Peking, eine umfassende Verwaltungs- und Gebietsreform durchgeführt. Das unter der Verwaltungshoheit Shanghais stehende ländliche Gebiet wurde drastisch erweitert zu einer administrativen Stadtregion. Die Stadt sicherte sich die Kontrolle über ihr Hinterland - auch, um ihren Bedarf an* industrieverwertbaren Rohstoffen, Wasser, Nahrungsmitteln und stadtnahen Güterumschlagsplätzen zu decken. Die tatsächlichen Gebietserweiterungen wurden in mehreren Schritten vollzogen. ${ }^{7}$ Das einheitlich verwaltete Stadtgebiet erweiterte sich von $636,18 \mathrm{~km}^{2}$ (1949) auf $5910 \mathrm{~km}^{2}$ (Dez. 1958). ${ }^{8}$

Die neue Satellitenstadt Minhang, ca. 1960

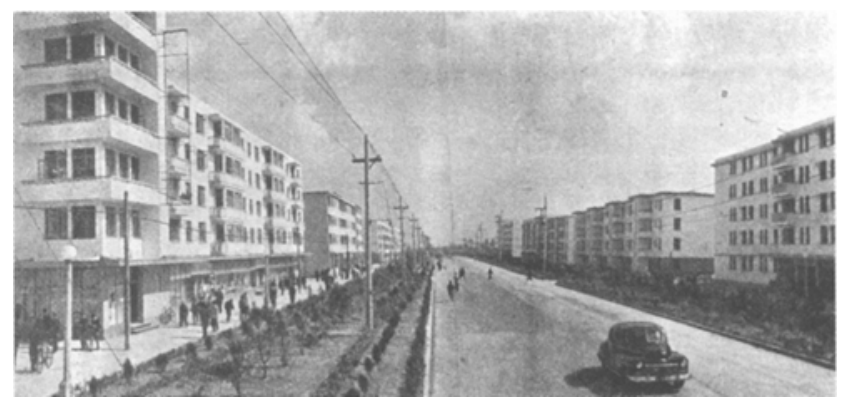

Quelle: Archiv Kaltenbrunner

Zum anderen betrieb die Stadt von nun an eine aktive stadt- und regionalplanerische Entlastungspolitik. Unter der Leitlinie „mehr, schneller, besser, wirtschaftlicher" (duo kuai hao sheng) wurde anlässlich der zweiten Sitzung der ersten Delegiertenkonferenz der KP Shanghai im Dezember 1957 beschlossen, im Umland der Stadt Satelliten zu gründen, um einen Teil der Industriebetriebe auf die Stadtregion zu verteilen und die Bevölkerungskonzentration im Kernbereich $\mathrm{zu}$ verringern. Ausgesprochen wurde der Wunsch, die Shanghaier Einwohnerzahl solle sich bei der Sieben-Millionen-Grenze einpendeln - eine Grenze, die zu diesem Zeitpunkt bereits erreicht bzw. überschritten war. ${ }^{9}$ Vorbereitende Untersuchungen zum neuen Generalentwicklungsplan waren soeben abgeschlossen worden; die allgemein anvisierten Planungsprinzipien für die Satelliten beruhten auf mehr oder minder ausgefeilten Studien. ${ }^{10}$ Dafür gab es historisch gesehen gute Voraussetzungen, wegen der traditionell sehr starken Vernetzung (in Form eines funktionierender Handelsaustauschs) zwischen Stadt und 
Land im Mündungsgebiet des Yangzi, die hier derart eng in- und miteinander verflochten waren, dass das eine ohne das andere kaum denkbar schien. Prozessuale und strukturelle Arbeitsteilung ist hier seit alters her Vorbedingung der wirtschaftlichen - und damit gesellschaftlichen - Prosperität. So konstatiert ein Beobachter einen „quasi-urbanen Charakter der Landschaft“; obwohl ihre Hauptfunktionen immer noch landwirtschaftliche seien, wäre doch der Modus ihrer Organisation industriell. ${ }^{11}$ Ihren hervorragendsten Niederschlag fand diese Planungspolitik in Minhang, in der ca. $30 \mathrm{~km}$ südlich des Shanghaier Zentrums am Oberlauf des Huangpu gelegenen, ersten chinesischen Satellitenstadt, die ab 1958 vehement entwickelt wurde.Der zentrale Baustein darin war die "Nachbarschaft" als Siedlungseinheit, die in China als sozialräumliche Organisationsform der Danwei eine kongeniale, autochthone Entsprechung fand. ${ }^{12}$

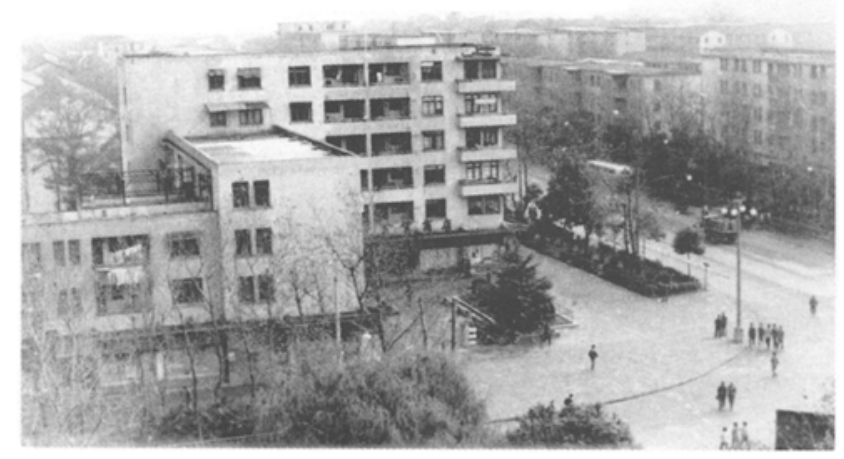

Foto: R. Kaltenbrunner 1985

Mit etwas Abstand gesehen war diese Planung ein Kompromiss zwischen widerstreitenden Interessen; und sie steht für den ambitionierten Versuch einer Industrialisierung ohne Verstädterung. ${ }^{13}$

Dabei sind nun aber mehrere Tiefenschichten interessant:

- Insgesamt ist diese Shanghaier Dezentralisierungspolitik als Programm zu begreifen, in dem Endogenes und Exogenes zusammenflossen und etwas Neues ergaben. Starke Wurzeln liegen in Ebenezer Howards Gartenstadt-Konzept mit seinen lebensund gesellschaftsreformerischen Inhalten; dies erfährt über diverse historische und gesellschaftspolitische Stränge eine eigenständige chinesische Implementierung. ${ }^{14}$
- Es war die Genese einer wissenschaftlichen Disziplin; sie emanzipierte sich von der Architektur auf der einen wie von der Wirtschaftsplanung auf der anderen Seite. Gleichsam als ReferenzmQdell wirksam war dabei das etablierte deutsche Stadtplanungssystem. ${ }^{15}$

- Und es gibt geheime Verbindungslinien namentlich zum Nachbarschaftskonzept eines C.A. Perry ${ }^{16}$, das seine hohe Zeit zwar in der unmittelbaren Nachkriegszeit hatte ${ }^{17}$, aber auch heute wieder ein Rolle spielt - (abstrakt) im Gewand von Kommunitarismus oder (ganz konkret) in den "gated communities".

- Die These von einer autozentrierten Entwicklung in China ist zu diesem Zeitpunkt bereits nicht mehr haltbar. ${ }^{18}$

\section{Atopische Ordnungsvorstellungen und Projekte}

Dem Betätigungsfeld „Satellitenstadt“ mit seinen aktiven Planungserfordernissen war jedoch keine lange Zukunft beschieden. In den Jahren nach 1961 - die Fehlentwicklungen und Katastrophen des „Großen Sprungs nach vorn" mussten kompensiert werden - fehlte es diesbezüglich an Kapitalzufluss. Das Aktionsfeld verlagerte sich, nicht nur in Shanghai, auf eine gänzlich andersgeartete, sozusagen abstraktere Ebene. In Einklang mit der reinen Lehre nach Marx waren „Konsumentenstädte“ in solche der „Produzenten“ umzubauen. ${ }^{19}$ Mit der Einführung von Volkskommunen ${ }^{20}$ hatten die alten „Leitbilder“ der Planer ihre Orientierungs- und Legitimationsfunktion weitgehend verloren. Gewohnte Vorstellungen und Verfahren versagten angesichts neuer Probleme, die nicht nur durch Fakten geschaffen, sondern auch durch Kursschwankungen innerhalb der Partei bestimmt wurden. ${ }^{21}$ Im dann folgenden gesellschaftspolitischen Umbruch der Kulturrevolution erlebte die Stadt- und Regionalplanung eine weitgehende Entkonzeptionalisierung. Nun ging es um ,atopische“ Ordnungsvorstellungen. Größere Maßnahmen wurden in Form sog. „Massenbewegungen“ (d.h. Arbeitseinsätze) mittels politischer Agitation realisiert. Und die Planung hatte ihren Raumbezug weitgehend aufgegeben.

Zu einer grundsätzlichen Neubewertung konzeptioneller Stadt- und Regionalplanung kam es erst wieder ab 1978 / 80 im Zuge der wirtschaftspolitischen Readjustierung. Erste Ansätze einer Stadterneuerung (häufig allerdings in Form einer Kahlschlagsanierung) sowie die Wiederaufnahme der Dezentralisierungspolitik (neue Satellitenstadt Jiading) stellten die beiden Grundlinien der Stadtplanung jener Dekade dar. 


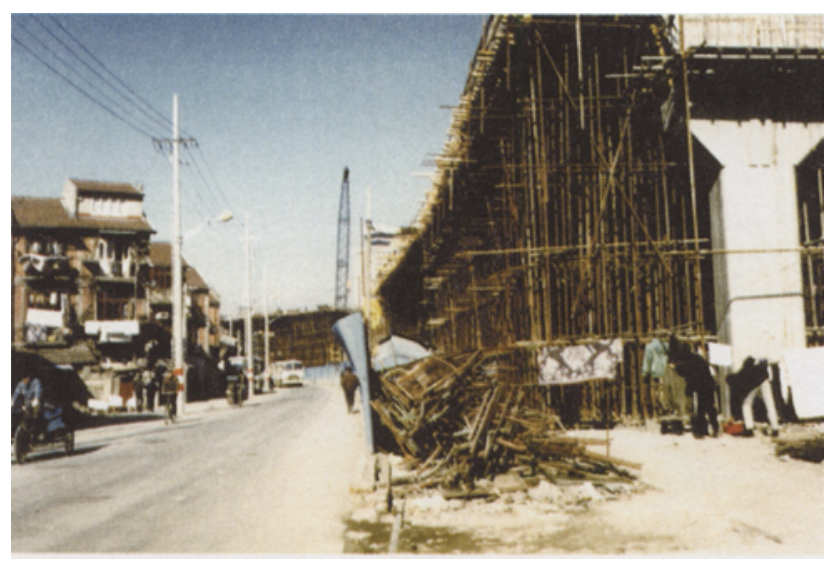

Bau der neuen Hochstraße im westlichen Stadtzentrum Shanghais Foto: R. Kaltenbrunner 1995

Man geht wohl kaum zu weit, dies als eine Phase der Reetablierung und Selbstvergewisserung der Disziplin $z u$ deuten. Man knüpfte an die theoretischen Positionen und Debatten der 50er Jahre an, wobei eine wichtige, gleichsam kulturphilosophische Linie eines eigenständigen chinesischen Entwicklungswegs auszumachen ist, die bis zu Kang Youwei und Liang Qichao zurückreicht, den führenden Intellektuellen der Bewegung vom 4. Mai 1919. ${ }^{22}$

In der Rückschau war dies jedoch nur eine Art Zwischenhoch: Denn etwa zeitgleich, als sich in Deutschland im Zeichen der Wiedervereinigung ein Umbruch vollzog, änderten sich auch in China um 1990 mit den Special Ecomomic Zones $^{23}$ die Parameter der Planung. Diesen Programmschritt hin zu einer "sozialistischen Marktwirtschaft" öffnete nicht nur die Tür für westliche Technologie, Zivilisation und Kapitalien, sondern zugleich den Himmel für Städte, deren Höhenrausch ihrem Flächenwachstum in nichts nachzustehen scheint. Obgleich - oder gerade weil - dieser Prozess durchaus im Einklang mit den Gesetzen eines post-fordistischen, globalen Kapitalismus steht, zeichnet sich in seiner Natur auch eine Neuskalierung des von Staat und Administration organisierten Raums ab.

Neben Shenzhen wurde Shanghai zum Motor der Öffnungspolitik: galt doch die wirtschaftliche Kraft der Yangzi-Metropole, die in seiner jüngeren Vergangenheit selbst durch gegenläufige Wirtschaftsprogramme nicht gebremst werden konnte, als schlagendes Argument für die Modernisierung. Deng Xiaopings berühmtes Wort, dass die Farbe der Katze gleichgültig sei, Hauptsache, sie fange Mäuse, wird nicht umsonst von Shanghaiern stolz auf sich selbst bezogen.

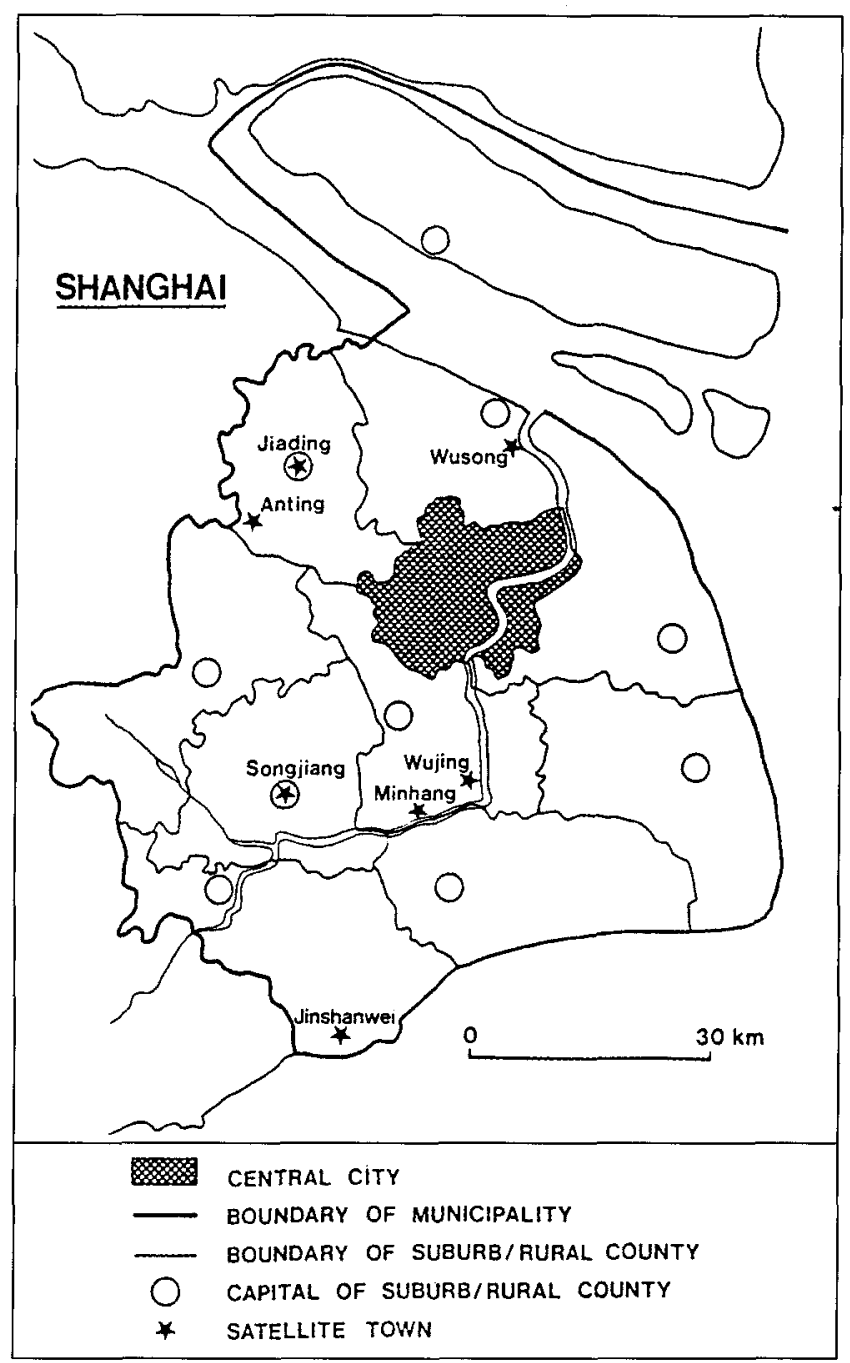

Shanghais Satellitenstädte mit „industriellem“ Schwerpunkt Planung und Bestand, ca. 1985

Quelle: Victor F.S. Sit (ed.) Chinese Cities - The Growth of the Metropolis since 1949. Hongkong - Oxford - New York 1983

Zwar bemüht die Stadtverwaltung sich augenscheinlich um eine gewisse Kontinuität in ihrer Planungspolitik: So werden erneut, in Form so genannter Wirtschaftssonderzonen, neue "Entlastungsstädte“ in der Stadtregion projektiert, ganz aktuell etwa die Hafenstadt Luchao ${ }^{24}$ oder die "Shanghai International Automobile City" in Anting westlich der Kernstadt. ${ }^{25}$

Aber der zentrale, alles überwölbende Entwicklungsschwerpunkt heißt seit geraumer Zeit Pudong, womit man, in modifizierter Form, wieder an den "Greater Shanghai Plan" anknüpft. Im wenig erschlossenen Osten der Stadt, jenseits des Huangpu, gelegen und auf einer Fläche von $350 \mathrm{~km}^{2}$ ausgewiesen, ist dies ein städtischer Investitionsbereich der Superlative. ${ }^{26}$ Die Gesamtkonzeption beinhaltet einen neuen Hafen 

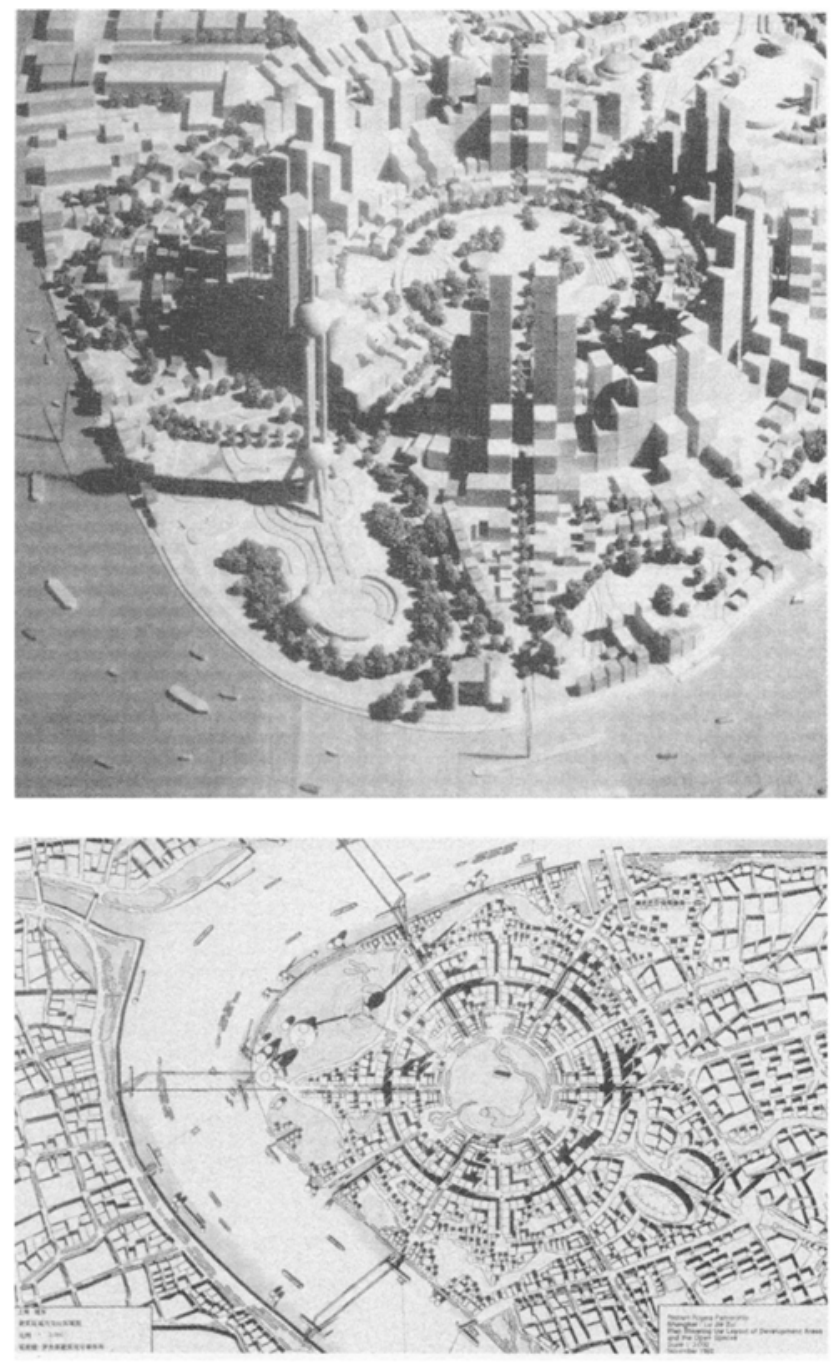

Das neue Zentrum Lujiazui in Pudong nach Plänen von Richard Rogers (1992)

Quelle: Der Architekt Nr. 3/1993

(lokalisiert im Bereich Waigaoqiao), einen weit östlich des Huangpu situierten Zentralflughafen (ein neuzeitliches Äquivalent zum Bahnhof von Jiangwan $)^{27}$ sowie ein neues Wohn-, Arbeits- und LebensZentrums für eine halbe Million Einwohner in Lujiazui. Die hierfür ausgearbeiteten Planungen des britischen Star-Architekten Richard Rogers ${ }^{28}$ allerdings sind später wieder verworfen worden. Die von und in Pudong entfaltete Entwicklungsdynamik sprengt alle europäischen Vorstellungen. Aber selbst chinesische Kollegen weisen auf Defizite hin. ${ }^{29}$

Was lässt sich aus dieser Entwicklung herauslesen? Das sind insbesondere vier Punkte:

- Gesellschaftlich fundierte Theorien der Planung spielen keine nennenswerte Rolle mehr: Planung wird gleichsam der Domäne der Architektur einverleibt; man könnte auch (in Anlehnung an
Häußermann/Siebel) von einer "Festivalisierung der Stadtpolitik" sprechen, die eher Stadt-Marketing als Stadt-Planung darstellt.

- Das Ziel der Shanghaier Stadtregierung istes, in den Club der Global Cities aufgenommen zu werden; die Entfesselung dynamischer Marktkräfte will ihr Modernitätsbestreben bildhaft machen; dafür rekurriert man auf bekannte Symbole des Fortschritts; bevorzugte Topoi sind Wolkenkratzer und Skyline. Uneingestanden, aber doch deutlich, heißt das heimliche Modell Big Apple (New York). ${ }^{30}$

- Eine weitere Kontextbestimmung liegt in den „New Urban Waterfronts", insbesondere den Londoner Docklands, aber auch in den Stadtumbauprojekten von Barcelona über Saloniki bis Sydney.

- Es ist teilweise (und erstmalig) eine Preisgabe der staatlichen Dominanz von Planung zu verzeichnen (Joint Venture, diverse PPP-Modelle).

Es gibt aber auch Implikationen auf der Makroebene:

Die Politik der Sonderwirtschaftszonen ist - auf nationalem und regionalem Level - nichts anderes als Planung durch Projekte. Und damit ist man wieder an dem Punkt, von dem aus man so hoffnungsvoll aufgebrochen war: Der Küstenbereich boomt, aber nur der. Die Landesplanung zementiert also eine überkommene, koloniale Wirtschaftsstruktur. Was im Zeichen der Ausbeutung geschaffen worden war, erfährt eine "sozialistische "Bestätigung. ${ }^{31}$ Ist das nun Ausdruck eines chinesischen Nützlichkeitsdenkens, das allein auf kurzfristige Effekte zielt, zu Lasten einer ausgewogenen Entwicklung? Oder heißt das, dass die wirtschaftsgeographische Verteilung der Kolonialzeit so ,irrational" nicht war?

- Das Konzept der dezentralen Konzentration auf Landesplanungsebene und einer äquilibristischen Raum- und Gesellschaftsentwicklung ist obsolet und durch kein neues ersetzt worden.

- Es greift ein neuer Regionalismus Raum: Die Emanzipation von der Zentralregierung wie auch die Konkurrenz der Stadt-Regionen untereinander nimmt zu (Verteilungswettkampf um staatliche Ressourcen und politischen Einfluss). 


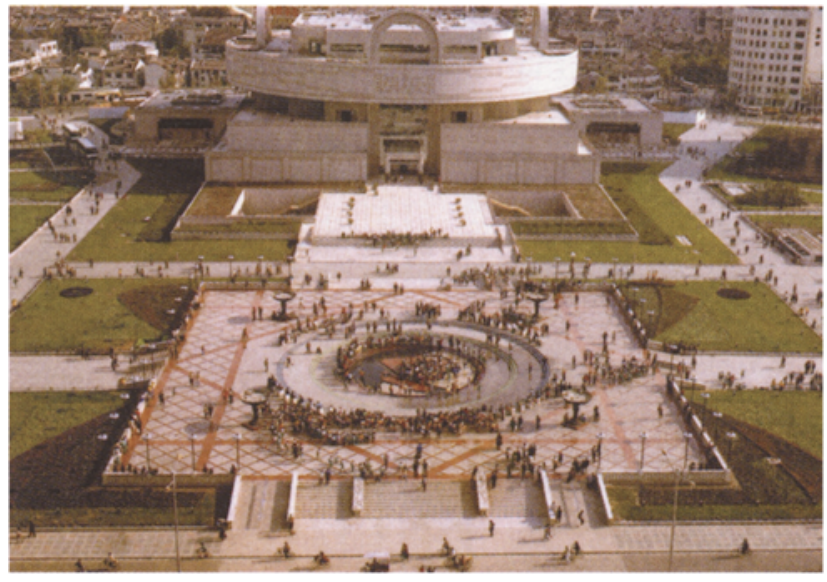

Das neue Kulturzentrum am „Peoples Square“, Shanghai Foto: R. Kaltenbrunner 1998

\section{Zwischenfazit}

Was hier angerissen wurde, ist zugegebenermaßen holzschnittartig und arg komprimiert. Deswegen ein kleines Zwischenfazit in drei Thesen:

These 1

Satellitenstädte wurden im Westen wie im Osten gebaut. Dezentrale Konzentration war hier wie da ein Thema. Nachbarschaftseinheiten erfreuten sich hüben wie drüben nahezu ungeteilter fachlicher Aufmerksamkeit. Wenn nun analoge Leitbilder bei Planern aus unterschiedlichen gesellschaftlichen Systemen und mit sogar entgegengesetzten politischen Ideologien $\mathrm{zu}$ finden sind, so kann dies auch als ein Indiz für den Praxisbezug und das Assimilationsvermögen stadtplanerischer Ideen interpretiert werden. Also ist es auch ein Zeichen dafür, dass den politischsozialen Unterschieden vergleichbare wirtschaftliche und siedlungsdynamische Bedingungen zu Grunde lagen, auf die die jeweils junge wissenschaftliche Disziplin Stadtplanung jeweils ähnlich reagierte.

\section{These 2}

Wir sind versucht, Geschichte als Abfolge von Phasen zu sehen. Deswegen nehmen wir Veränderungen oder Zäsuren deutlicher wahr als Kontinuitäten und Überlagerungen. Zentrale Aspekte dessen, was unsere Diskussion über Planung bestimmt, findet einen Widerschein in China: die angebliche Dichotomie von Theorie und Praxis, die Weichenstellung hin zu einer problemorientierten, gesellschaftsbezogenen Raumwissenschaft, verbunden mit dem Glauben an den normativen Charakter der Entwicklungsplanung, die jedoch ob ihrer Erfolglosigkeit oder Langfristigkeit einer „Planung durch Projekte“ Platz machen musste. Die planerischen Paradigmen in beiden Ländern sind einander nicht fremd und weisen eine ähnlich wechselvolle Geschichte auf.

\section{These 3}

Obgleich formell als "sozialistisch“ apostrophiert, mehren sich auch in der VR China strukturelle Anzeichen dessen, was Anthony Giddens „moderne Gesellschaft“, was Gerhard Schulze „Erlebnisgesellschaft“ oder Peter Gross „Multioptionsgesellschaft“ genannt haben. Das Spannungsfeld Standardisierung versus plurale Lebenstile, also harte Ökonomie versus gesellschaftliche Trends, wird bedeutsamer; Zeit-, Problemund Reaktionszyklen nähern sich einander an. Mit dem stärkeren Gewicht, das der Lebensqualität beigemessen wird, steigen die Ansprüche, und damit beispielsweise auch der Flächenverbrauch. Die StadtRegion, die Binnenstrukturen der Agglomerationsräume, die Umlandbeziehungen: das sind zunehmend die Aufgaben und Themen der Planung.

Hier schließt sich ein Kreis, weil es das konstituierende Motiv der Disziplin berührt: den Gegensatz von Stadt und Land, den es so nicht (mehr) gibt und den aufzuheben auch die sozialistischen Klassiker immer versprochen hatten. ${ }^{32}$

Auf das Hier und Heute übertragen, lassen sich aus dem Skizzierten durchaus einige Schlussfolgerungen ziehen. Zunächst einmal, und überspitzt ausgedrückt: Man ist wieder da, von wo aus man in der Planungseuphorie der 50er/60er Jahre (in China) bzw. der 60 er/70er (in Deutschland) aufgebrochen ist. Weit verbreitete Planungsfeindlichkeit aus ideologischer Voreingenommenheit und auf Grund partikularer Interessen (Neoliberalismus), Misstrauen gegenüber öffentlichen Maßnahmen und der so genannten Bürokratie, die Neigung, das Prinzip des freien Kräftespiels unterschiedslos auf private und öffentliche

Die neue Autobahnbrücke über den Huangpu, Shanghai Foto: R. Kaltenbrunner 1998

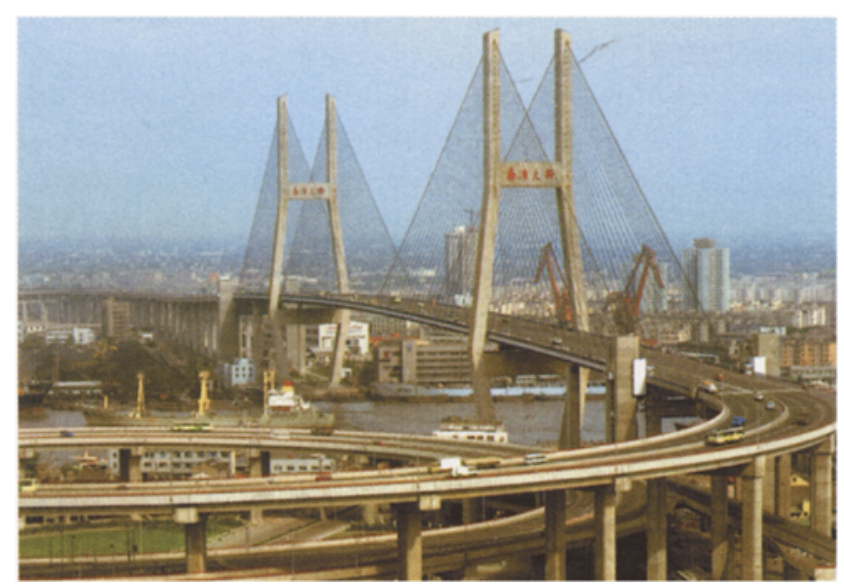


Angelegenheiten anzuwenden, ungenügende Kooperation von Marktkräften und öffentlichen Händen sowie mangelnde Einsichten von Politik, Wirtschaft und Öffentlichkeit in die Komplexität und Tragweite von Stadt- und Regionalplanung.

\section{Was heißt das fürs Nachdenken über Planung?}

Wenn dieser Befund stimmt, macht es dann noch Sinn, die Bedeutung von Leitbildern zu thematisieren? In einer erweiternden Einschätzung soll abschließend eine Antwort in fünf Punkten versucht werden:

(1)

Der gesellschaftliche Stand von Planung und Planungstheorie könnte besser sein. Mehr und mehr sind die Wirtschaftsförderung, das Stadtimage und die Verbesserung der Standortbedingungen ins Zentrum der Stadtpolitik gerückt. Eine politische Konsensbereitschaft über die baulich-räumlichen oder gesellschaftlichen Ziele der Stadtentwicklung gibt es allenfalls in Ansätzen. Ein zentrales Problem liegt sicherlich darin, dass die Bürger sich nur für Details ( sei es der Parkplatz oder die Fahrradspur vor der Tür, der Spielplatz um die Ecke oder die Revision der Hundeverordnung) interessieren, das aber dann vehement (die Erfolgsgeschichte der Bürgerinitiativen ist bekannt!). Bislang kennt man in Shanghai so gut wie keine partizipativen Ansätze der Planung. ${ }^{33}$ Wenn aber bei uns das Paradigma der Bürger und Planungsbetroffenen in der Regel „Nimby“"34 lautet, muss man sich die Frage stellen, wie „bürgerschaftliche Teilhabe“, Subsidiarität, „Verantwortung vor Ort“ glaubhaft - und d.h. anders denn als lokale Egoismen oder Verhinderungsstrategien - als Leitgedanken reintegriert werden können.

(2)

Das, was als „aufgeklärte“, theoretisch fundierte Planung bezeichnet werden könnte, weist aus einem bestimmten Blickwinkel blinde Stellen auf. Ihre Maxime lautet, knapp gesagt: "Stadt" lasse sich heute weniger denn je von einem Leitbild, einer bildhaft in die Zukunft projizierten Form her konzipieren, sondern entstehe eher induktiv aus dem komplexen Mit- und Gegeneinander der städtischen Akteure. Es ist jedoch gleichsam paradox, wenn umgekehrt über den Verlust einer grundsätzlichen und integrierten Gesamtplanung lamentiert und das Peu à Peu von Stadtteilentwicklung, der Inkrementalismus und der quasi allmächtige Projektbezug kritisiert wird. Es geht weniger darum, alte Muster oder Theorien der Planung durch gänzlich neue, modernere zu ersetzen, sondern um ihre sinnvolle und intelligente Kombination. ${ }^{35}$

\section{(3)}

Der sicherlich vorhandene Konflikt zwischen Froschperspektive und Vogelschau, d.h. der „Bilderwelt der Architekten“ und der - regelmäßig ernüchternde planungstheoretische Versuch einer Thematisierung von „Prozessstrukturen, gesellschaftlichen Zusammenhängen, politischen und Entscheidungsgesetzmäßigkeiten, von prozessualem und strategischem Denken" ${ }^{36}$ darf nicht zum Krieg ausarten, sondern muss - wie auch immer - fruchtbar, wenn möglich zu einer Art Dialog gemacht werden.

\section{(4)}

Die kommende Generation von Stadt- und Regionalplanern sollte in der Lage sein, rasch und überzeugend Ziele zu entwerfen, flexibel zu handeln, ohne die städtebaulichen und gesellschaftlichen Qualitätsansprüche zu vernachlässigen. Und sie sollte über eine überdurchschnittliche kommunalpolitische Sensibilität verfügen. Das Erkennen und Nutzen von Zeitund Politikfenstern wird somit genauso wichtig wie die Fähigkeit zur Analyse. Interkultureller Austausch ist dafür heute unverzichtbar. Insofern ist auch die Trennung von Theorie und Empirie, von Wissenschaft und Praxis fragwürdig - das ist kein Gegensatz, sondern eine Ergänzung.

(5)

Die besondere Dialektik der Profession offenbart sich im Methodischen: Zugleich offen zu halten und festzulegen; neuen Entwicklungen und dem gesellschaftlichen Wandel neue Möglichkeiten bieten, ohne unverbindlich zu werden. Dafür angemessene Methoden zu finden, dürfte eine der wichtigsten Aufgaben sein. Verhandlung und Kooperation sind zentrale Stichworte. (Wobei jedoch die öffentliche Hand gut beraten ist, das überlieferte Instrumentariums zwar dosiert, dann aber auch bewusst und zielgerichtet einzusetzen. ${ }^{37}$ ) Planung heute ist eine immer wieder neue Mischung aus Konzeption und Moderation. Lösungen werden zwischen den Logiken von Staat und Markt liegen, wofür u.U. „informelle Planungen“ das probate(re) Mittel darstellen.

Die neue Stadtsilhouette des Shanghaier Zentrums

Foto: R. Kaltenbrunner, 1998

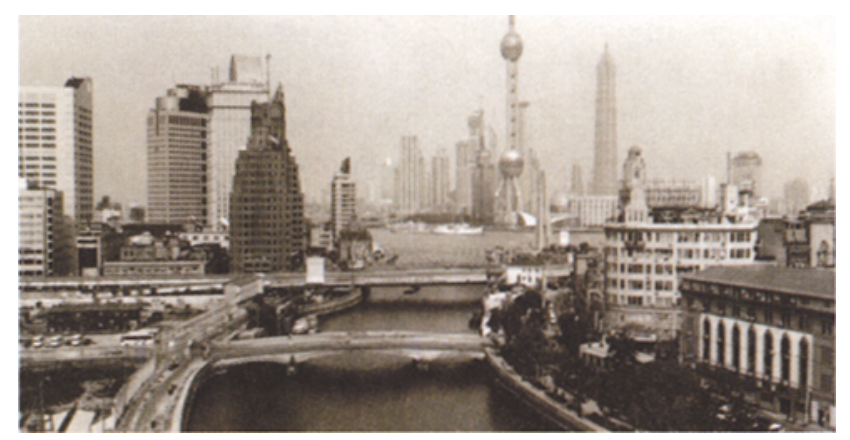




\section{Trotzdem - Leitbilder sind nicht obsolet}

Paradigmen der Planung sind, namentlich im Bezug auf Großstadtregionen in den letzten 75 Jahren, eingebettet in quasi überzeitliche Zusammenhänge. Indem der "Statik des Gebauten“ in immer größerem Ausmaß eine „Dynamik des städtischen Lebens" (H.P. Bahrdt) gegenüberzusetzen war, mussten die Baustrukturen der Zentren zur Disposition gestellt und den Potenzialen der städtischen Peripherien eine stärkere Aufmerksamkeit gezollt werden. Objektivierbare Zahlen wurden zum tertium comparationis der verschiedenen Interessen und Ansprüche. Die einprägsamen Bilder bestimmter städtebaulicher Konfigurationen galten lange Zeit nicht mehr; heute jedoch scheinen sie erneut eine Verständigungsgrundlage zwischen den Disziplinen Politik, Wirtschaft, Raum- und Fachplanung darzustellen. Die explosiven Entwicklungen in Produktion, Demographie, Handel und Verkehr forderten ständig neue Konzepte (und erzwangen mitunter eine Rückkehr zu alten). Es bleibt festzuhalten, dass Shanghai solche formuliert hat - wenngleich deren Ergebnisse der Metropole nicht immer zum Vorteil gereichten.

Gemessen an den Ansprüchen eines je umfassenden und wie auch immer gearteten Leitbildes offenbart sich in vielen Planungen eine unvollkommene, ja stecken gebliebene Transformation. Prozessual gesehen sind jedoch die entstandenen Gebilde (Städte, Regionen) folgerichtige Ergebnisse eines komplexen, zeittypischen Kräftespiels und insofern ein getreuer Spiegel der jeweiligen struktur- und gestaltbestimmenden gesellschaftlichen und wirtschaftlichen Verhältnisse. Die Geschichte lehrt, dass der rasche Wandel von Rahmenbedingungen und Aufgabenschwerpunkte, mehr aber noch die Verschiebungen in den Wertsetzungen, also der Mangel an Kontinuität in der Formulierung von (Nah-)Zielen und Strategien, zu rudimentären Realisierungen und partiellen Verformungen führten.

Klar ist: Planung erfordert Kompromisse, und zwar permanent. Indes: Allen dargestellten Ambivalenzen zum Trotz - oder gerade deswegen - braucht Planung auch weiterhin Leitbilder. Wie auch immer ein solches formuliert oder ausgerichtet sein mag: Es ist ihr unabdingbarer Zielhorizont, ihr reflexives Rückgrat. Und als solches ist es letztlich jeder Planung, die diesen Namen verdient, immanent.

\section{Anmerkungen}

(1)

In diesem Zusammenhang sei nur auf den voluminösen Band "Ohne Leitbild? Städtebau in Deutschland und Europa" hingewiesen, den Jens Jessen, Heidede Becker und Robert Sander 1996 herausgegeben haben.

(2)

Vgl. Wu, T'ieh-ch'eng: „Greater Shanghai - its present and future“. People's Tribune, 3, 1933, S. 400-405

(3)

Vgl. Robert Kaltenbrunner: „Shanghai's architectural heritage: Housing developments in the 1920s and 1930s". EKISTICS. The problems and science of human settlements, 58, 346/347, 1991, S. $89 \mathrm{ff}$.

(4)

Der Verweis auf die Pariser Schule der Beaux Arts ist überdeutlich. Die Formensprache wirkt wie klassischen Beispielen aus Griechenland oder Weltmetropolen wie Rom und Paris entnommen: Vistas, Boulevards, Fontänen, Terrassen, Statuen und Kolonnaden gestalteten den städtebaulichen Raum wie Sonntagskleider.

(5)

Es ging also um den Aus- bzw. Aufbau weit im Inland gelegener neuer Ansiedlungen, die dem Aspekt einer ausbalancierten, äquilibristischen Raumordnung gehorchten, um starke regionale Disparitäten auszugleichen. Die wichtigsten Schwerpunktstädte waren Lanzhou, Xian, Luoyang und Wuhan, Chengdu, Taiyuan und Datong, Jilin sowie Anshan. Diese Politik, bei einem begrenzten Investitionsvolumen eine hohe Konzentration der Mittel auf wenige Schwerpunkte zu verfolgen, war innerhalb der Partei jedoch -wegen der damit verbundenen Finanzierungsprobleme - nicht unumstritten.

Wenn trotz unterschiedlicher Voraussetzungen in China das sowjetische Planungssystem anfangs vielleicht relativ unreflektiert übernommen wurde, so erklärt sich die Attraktivität dieses Modells zum einen sicherlich daraus, dass es bis dahin das einzige historisch erprobte System einer Planwirtschaft war und als solches auch bereits durchaus Erfolge vorweisen konnte. Andererseits galten die Schlüsselargumente, die für ein hochzentralisiertes Planungssystem in der sowjetischen Industrialisierung angeführt wurden, natürlich auch für China: die Notwendigkeit des beschleunigten Aufbaus einer eigenen schwerindustriellen Basis, um sich von außenpolitischen Abhängigkeiten so weit wie möglich frei zu machen; die relativ einfache und überschaubare Produktionsstruktur der vorhandenen, unterentwickelten Wirtschaft; die räumlichen Bedingungen eines riesigen Landes, d.h. die damit verbundenen Investitions-, Transportund Arbeitseinsatzprobleme. Lenins Internationalismus verlieh der sowjetischen Konzeption eines zentral-administrativen Planungssystems eine bestätigende Plausibilität. In diesem Zusammenhang kam den von der UdSSR entsandten „Beratern“, die dem "Modell“ praktische Gestalt verliehen, ein nicht zu unterschätzender Einfluss zu (vgl. Franz Schurmann: Ideology and Organization in Communist China. Berkeley, Los Angeles 1969, S. $180 \mathrm{f}$.$) .$ 
(6)

Jüngst, Peter; Peisert, Christoph; Schulze-Göbel, Hans-Jörg (Hrsg.): Stadtplanung in der Volksrepublik China. Entwicklungstrends im Spiegel von Aufsätzen und Gesprächen (1949-1979). Reihe Urbs et Regio, Bd. 35. - Kassel 1984, S. 101

(7)

Ein vorläufiges Ende fand sie 1962 mit dem 10. Plenum des Zentralkomitees, in dem der Beschluss gefasst wurde, Städte mit ihrem Umland zu so genannten Wirtschaftsgebieten (jing ji qu yu) zusammenzufassen.

(8)

Vgl. Yan Zhongmin: „Shanghai: The Growth and Shifting Emphasis of China's Largest City“. In: Sit, Victor F.S. (ed.): Chinese Cities - The Growth of the Metropolis since 1949. Hongkong, Oxford, New York 1988, S. 113 f.

(9)

Gemeint ist hier die Bevölkerung der City und der unmittelbaren Stadtrandzonen. Man muss sich die Daten des Wachstums vergegenwärtigen, um diesen Druck nachvollziehbar zu machen: 1949 beherbergte das Stadtgebiet ca. 4,5-5 Mio. Menschen. Schon 1953 war diese Zahl auf 6,2 Mio. gestiegen. Unter Zuhilfenahme eines Rücksendeprogramms (xia fang) wurden 1955 etwa 500000 ländliche Migranten aus der Stadt gelotst - deswegen sei die Einwohnerzahl 1956 nur knapp über der 6-Millionen-Grenze gelegen. Durch einen Aufwärtstrend in der Geburtenrate seit Mitte der 50er Jahre und der Landflucht von ca. 750000 Bauern und Landarbeitern als Ergebnis der Kollektivierungskampagnen in der Agrarwirtschaft und dem deutlich angehobenen (städtischen) Industriearbeiterlohn stieg Shanghais städtische Bevölkerung auf rund 7,2 Mio. Ende 1957 an. Der Bevölkerungszuwachs, für die $50 \mathrm{er}$ Jahre im Durchschnitt bei $4 \%$ anzusetzen, übte zwangsläufig einen gewaltigen Druck auf die sowieso schon völlig überlastete städtische Infrastruktur aus. Insbesondere gestaltete sich die Wohnungsfrage zunehmend problematischer: Die Pro-Kopf-Wohnfläche in Shanghai war 1957 wesentlich niedriger als zu Beginn der 50er Jahre. Vgl. hierzu ausführlich Kirkby, Richard J.R.: „A Review of Satellite Town Policies in the People's Republic of China: The Experience of Shanghai“. In: Phillips, David D.; Yeh, Anthony G.O. (Hrsg.). New Towns in East and Southeast Asia: Planning and Development. Hongkong, Oxford $\mathrm{u}$. New York, 1988, S. 212ff.; sowie Howe, Christopher: "The Supply and Administration of Urban Housing in Mainland China: The Case of Shanghai“. China Quarterly, 33: 1968, S. $78 \mathrm{ff}$.

(10)

Vgl. Qian Shengtie: „Zur Planung von Satellitenstädten im Raum Shanghai“. In: Jüngst et.al., S. 105-123. (Original: Jianzhu Xueb$a o, 8,1958$, S. 30-33)

(11)

White, Lynn T. III: „Shanghai - suburb relations, 1949-1966“. In: Howe, Christopher (ed.). 1981. Shanghai. Revolution and Development in an Asian Metropolis. Cambridge University Press, 1981, S. 259. Vgl. auch Elvin, Mark: „Market towns and waterways: the county of Shanghai from 1480 to 1910 ". In: Skinner, G.W. (ed.): The City in Late Imperial China, Stanford, 1977, S. $441-473$
(12)

Der missverständliche Begriff „Nachbarschaft" spielte bei Standort- und Inhaltsbestimmung in der internationalen Planungsdiskussion eine wichtige Rolle; und die Wohngebietsplanung auf der sozialräumlichen Basis der Danwei stellt gleichsăm eine neue Art von Zugeständnis an die gesellschaftliche Wirklichkeit der VR China dar. „Danwei“ heißt wörtlich übersetzt „Grundeinheit". Sie ist zentraler Baustein für eine Art zellulare Organisation der gesamten chinesischen Gesellschaft, die viel an Sicherheit und Geborgenheit bot, aber auch mit einer quasi absoluten sozialen Kontrolle einherging. Sie war über Jahrzehnte hinweg umfassend und allgegenwärtig in Chinas Städten, verlor indes im Zuge der wirtschaftspolitischen Liberalisierung in den 90er Jahren zunehmend an Bedeutung. Vgl. u.a. Lü Xiaobao u. Elizabeth J. Perry (eds.): „Danwei. The Changing Chinese Workplace in Historical and Cooperative Pespective". New York u. London 1997

Vgl. hierzu auch Koshizawa, Akira: "China's Urban Planning: Toward Development Without Urbanization". The Developing Economies, 16, 1, 1978, S. 3-33

\section{(14)}

In diesem Zusammenhang ist sowohl auf diverse sowjetische Planungskonzeptionen der 20er Jahre wie auch den Reformwohnungsbau der Weimarer Republik, namentlich von Ernst May in Frankfurt a.M., zu verweisen. Insbesondere spielten aber auch Patrick Abercrombies „Greater London Plan“ von 1944 und die britische New Town Policy der unmittelbaren Nachkriegszeit eine wichtige Rolle.

\section{(15)}

Insbesondere die enge Verzahnung von Ingenieurtechnik, Rechts- und Bodensystem, Kommunalpolitik usw. - bewundert wurde also im Grunde seine durchsetzende Effizienz.

(16)

Perry, Clarence Arthur: "The Neighborhood Unit. A Scheme of Arrangement for the Family-Life Community“. Regional Survey of New York and its Environs. Vol. III. New York, 1929 (Neudruck 1974). Prägend für Perry waren die Arbeiten bzw. Untersuchungsergebnisse der "Chicago School of Social Ecology“ um Park, Burgess und McKenzie (Park, Robert E.; Burgess, E.W.; McKenzie, R.D. The City. Chicago 1925).

\section{(17)}

In Deutschland etwa im Konzept der "gegliederten und aufgelockerten Stadt" von Göderitz/Rainer/Hoffmann

(18)

Vielleicht darf man von einer gewissen Globalisierung des Metiers, seiner Ideen und theoretischen Ansätze, sprechen.

(19)

Die Parole von der "Umwandlung der Konsumenten - in Produzentenstädte" (bian xiaofei chengshi wei shengchan chengshi) hatte als oberste Maxime für alle stadtentwicklungsbestimmende Arbeit bis ca. 1980 unumschränkte Gültigkeit. Erst mit der wirtschaftlichen Readjustierung, den zunehmend auftretenden Umweltproblemen im Gefolge und direkten Zusammenhang mit den letztlich strikt industriefördernden Maßnahmen und der 
grundsätzlichen Neubewertung konzeptioneller Stadtplanung zeichnete sich eine Wende ab (vgl. u.a. Buck, David D.: „Changes in Chinese Urban Planning since 1976“. Third World Planning Review, 6, 1, 1984, S. 5-26). Bereits vor der endgültigen Machtübernahme der KP, auf der zweiten Plenartagung des vom siebten Parteitag gewählten ZKs am 5. März 1949 in der Provinz Hebei, legte Mao in einer Ansprache die in dem Schlagwort zusammengefassten Grundzüge der späteren Politik gegenüber den Städten dar.

\section{(20)}

Die Volkskommunen waren als eine Art von Gemeinwesen entworfen, als Militär-, Produktions- und Verwaltungseinheiten mit eigenen Fabriken, Werkstätten, Schulen und Hospitälern; sie waren soziale Experimentierfelder, auf denen die „Eigentumslosigkeit" und die rationelle Ausnutzung der Arbeitskraft des Einzelnen zu Gunsten des Kollektivs gradweise erprobt wurde. Es gab radikale Formen der Verwirklichung, solche mit Gemeinschaftsbaracken und Kantinen, in denen die Löhne überhaupt abgeschafft waren, und andere mit Halbversorgungssystem, Halblohn und Familienwohnung. Doch der Elan, mit dem man begonnen oder den die Propaganda vorgetäuscht hatte, verflog rasch. Völlig gescheitert war der Versuch, primitive Kleintechnologien und -industrien auf Dorfbasis zu errichten (die „Hinterhof-Schmelzöfen“), denn der technische Rückstand machte ihre Produkte kostspielig und unbrauchbar. Nach der schweren Wirtschaftskrise der Jahre 1960/61 blies die Partei zum Rückzug; das Experiment wurde abgebrochen, die Volkskommunen nur noch als Verwaltungseinheiten verstanden. Das revolutionäre Paradoxon, das Mao Zedong kurz vor dem Großen Sprung verkündet hatte, dass nämlich China gerade wegen seiner Armut und Rückständigkeit und wegen seiner gigantischen (und ständig steigenden) Bevölkerungszahl in kurzer Zeit die kapitalistischen Länder (wirtschaftlich) einholen könne, hatte sich selbst widerlegt. Auch die Parteiideologen mussten eingestehen, dass die egalitäre Utopie des "Jeden nach seinen Bedürfnissen“ noch nicht verwirklicht werden konnte, sicher jedenfalls nicht im Großen Sprung.

Vgl. Buck, David D.: „Directions in Chinese Urban Planning“. Urbanism Past and Present, 1, 1, 1976, S. 29; sowie Pannell, Clifton W.; Ma, Laurence J.C.: China - The Geography of Development and Modernization. London. 1983, S. 233

(22)

Wichtige Fixpunkte dieser Linie sind Liang Sicheng (der Sohn Liang Qichaos) als der zentrale Architektur- und Stadttheoretiker Chinas der 50er Jahre (später in der Kulturrevolution umgekommen), sowie dessen „Schüler“ Wu Liangyong und Hou Renzhi in den 80er Jahren.

Im Februar 1984 steckte Deng Xiaoping in dürren Worten das entsprechende Experimentierfeld ab: „Wir sollten erstens bei den Bereichen Transport und Kommunikation beginnen, da das der Ausgangspunkt für jede wirtschaftliche Entwicklung ist. Zweitens sollten wir uns für eine Politik entscheiden, die hohes Einkommen und hohen Konsum fördert. Da entsprechende Bedingungen noch nicht im ganzen Land vorhanden sind, können wir einige Gebiete 'als erste reich werden lassen'. Egalitarismus wäre fehl am Platz." Zit. n. Deng Xioaping: Die Reform der Revolution. Eine Milliarde Menschen auf dem Weg. Berlin 1988, S. $49 \mathrm{f}$.
Diese Wirtschaftsonderzonen sollten durch ein spezielles Angebot ausländisches Kapital anziehen sowie die chinesische Exportwirtschaft fördern. So werden Anlegern für Gemeinschaftsunternehmen (joint ventures) Steuererleichterungen gewährt, beispielsweise eine Körperschaftsteuer, die unter den ent sprechenden Sätzen von Hongkong und Taiwan liegt. Weitere Investitionsvorteile sind Zollerlass und das Recht, Arbeitskräfte einzustellen und zu entlassen, sowie die Freiheit, Außenhandelsverträge zu schließen. Physisch hat man sich unter einer solchen Zone ein gut erschlossenes Territorium vorzustellen, vom Umland durch Zäune, Mauern und eigene Polizeiwachen getrennt, der Zugang streng kontrolliert wie an einer Landesgrenze. Das ist denn der Ort, an dem mit „Formen der Marktwirtschaft experimentiert" wird, wie man die stattfindenden Prozesse beschönigend umschreibt.

\section{(24)}

Die neue Hafenstadt Luchao für 300000 Einwohner, $60 \mathrm{~km}$ von der Kernstadt Shanghai entfernt, soll nach einem Entwurf des Hamburger Büros von Gerkan, Marg und Partner (gmp) gebaut werden. Das erste Drittel soll bereits 2005 stehen, 2020 könnte die ganze Stadt fertig sein. Sie entsteht zum Teil auf künstlich aufgeschüttetem Gelände um einen kreisrunden Süßwassersee von zwei Kilometern Durchmesser. Zwischen dem Geschäftszentrum und den äußeren Wohnquartieren verlaufen Ringstraßen und ein Grüngürtel.

\section{(25)}

Hinter dieser Entwicklung steht der Volkswagenkonzern, der eine „German Town mit Zentrum und Kirche“ für rund 60000 Einwohner auf der Basis deutscher Planungen vorsieht - vgl. IzR 4/5, 2001, S. 237 ff. Der für dieses Konzept verantwortliche Frankfurter Stadtplaner Albert Speer hat darüber hinaus im Rahmen des UIA-Weltkongresses (Juli 2002 in Berlin) ein „ökologisches Stadtbausystem" für Shanghai vorgestellt, das "fast ohne Emissionen“ arbeitet. Konzipiert werden "europäische“ Siedlungsstrukturen mit „chinesischem Inhalt“. Die modular erbauten Stadtquartiere von 400 mal 400 Metern (die alte Maßeinheit Li) bestehen aus sechs- bis achtgeschossigen Gebäuden und sind ausgestattet mit traditionellen Ladenstraßen und Stadtteilparks. Grüne Innenhöfe sollen „urbane Landwirtschaft“ ermöglichen. Ökologische, recycelbare Baustoffe und die Nutzung von Solarenergie und klimatischen Gegebenheiten minimieren den Ressourcenverbrauch. Den Verkehr sollen Elektrobusse und Privatautos bestreiten, die im Sinne eines Car-Sharings mehrfach genutzt und in automatisierten Parkregalen abgestellt werden. Inwieweit das reale Zukunftsaussichten sind, sei dahingestellt.

Vgl. auch Schmidt, Helmut: „Perestrojka auf chinesisch“. Die Zeit, 25, 1993, S. 11

(27)

Vgl. Cheng, Elisabeth: „Shanghai surprise“. Far Eastern Economic Review, 18. Juni 1992, S.64 f.

Schon die Form der Direkt-Beauftragung durch den Oberbürgermeister spricht eine deutliche Sprache, was die politische Dimension der Planung anbelangt. 
(29)

Sie basieren auf diversen Gesprächen des Verfassers mit unterschiedlichen Planungsbeteiligten und lassen sich wie folgt zusammenfassen: Es gibt keinen richtige Makro-Plan, keine Koordinierung und Abstimmung auf Gesamtstadtebene. Jeder Bereich ist ausschließlich mit seinem eigenen Fortkommen beschäftigt.

(30)

Die Kritik könnte noch weiter gehen: Städtebau und Stadtentwicklung sind in der chinesischen Wirklichkeit zu Tragschichten reduziert worden. Bei dieser geht es vorrangig um Impulse für die Ökonomie, bei jenem um die Produktion von Bildern - und um die Partizipation an einer universalen Image- und Zeichenwelt. Unter diesen Umständen ist heute weniger die Raumpolitik (d.h. die Auseinandersetzung über Richtung und Form des Wachstums innerhalb von Städten und Regionen) als vielmehr der interregionale Wettbewerb (d.h. die Auseinandersetzung zwischen Städten und Regionen in der Konkurrenz um Kapitalinvestitionen) zur zentralen Kraft bei der Produktion von und Transformation der Stadt geworden.

$\mathrm{Zu}$ Aspekten des rein empirischen Befunds vgl. u.a. Gu Chaolin et al.: "Growth of New Designated Cities in China“. In: Chinese Geographical Science. Vol. 9, Nr. 2, 1999, S. 97-106

(32)

Was wiederum nicht ohne Ironie ist, dürfte dafür wohl doch eher der herrschende Neoliberalismus verantwortlich sein. In diesem Zusammenhang wäre auf Suburbia und seine aktuellen Theorien zu verweisen: Zwischenstadt, Edge Cities, The Sprawl, „Peripherie ist überall“ usw.
(33)

Allerdings hat jüngst in Peking - im Zusammenhang mit der Planung des Olympia-Parks für die Olympischen Spiele 2008 - eine Art von Bürgerbeteiligung stattgefunden: Die Stadtplanungsbehörde stellte alle eingereichten Projekte öffentlich aưs und räumte der Bevölkerung am 23. Juli die seltene Möglichkeit der Mitbestimmung ein. Hierbei bekam nach Angaben der Zeitung China Daily der Entwurf des in San Francisco ansässigen Büros Hideo Sasaki \& Associates, der auch schon von einer internationalen Jury den ersten Preis erhalten hatte, die Mehrzahl der Stimmen. (Bau-Netz-Meldung vom 31.7.2002)

\section{(34)}

Akronym für das englische "Not in My Backyard“, das unserem „St-Florians-Prinzip“ verwandt ist.

\section{(35)}

Vgl. u.a. Fingerhuth, Carl: „Die Gestalt der postmodernen Stadt“, Zürich 1996, S. 119

(36)

Vgl. hierzu - wie auch zu anderen diskutierten Aspekten - den anregenden Beitrag von Klaus Selle mit dem Titel „Alte und neue Planungskulturen“ im Sammelband „Stadt im Wandel - Planung im Umbruch“, hrsg. von Tilman Harlander. Stuttgart 1998, S. $45-65$

(37)

Vgl. u.a. Hartmut Gaßner et al: „Mediation: Verhandlung als Mittel der Konsensfindung bei Umweltstreitigkeiten“. Bonn 1992

Dr. Robert Kaltenbrunner

Bundesamt für Bauwesen und Raumordnung

Deichmanns Aue 31-37

53179 Bonn

E-Mail: Robert.Kaltenbrunner@bbr.bund.de 